\title{
La administración estratégica en la calidad de la industria del vestido de Puebla, México
}

DOI: https://doi.org/10.21158/01208160.n87.2019.2444

Fecha de recepción: 03 de octubre de 2018

Fecha de aprobación: 19 de noviembre de 2019

\author{
Mario Antonio Burguete-Garcia \\ Benemérita Universidad Autónoma de Puebla \\ mario_burguete@yahoo.com \\ Enrique Darío Romero y Cejudo \\ Benemérita Universidad Autónoma de Puebla \\ eromeroc@prodigy.com \\ Ramón Acle-Mena \\ Benemérita Universidad Autónoma de Puebla \\ raclemx@yahoo.com.mx \\ (c) $(\mathrm{P})($
}

Cómo citar este artículo / To reference this article / Comment citer cet article / Para citar este artigo:

Burguete-Garcia, M. A.; Romero y Cejudo, E. D.; Acle-Mena, R. (2019). La administración estratégica en la calidad de la industria del vestido de Puebla, México. Revista Escuela de Administración de Negocios, (87), 117-137. DOI: https://doi.org/10.21158/01208160. n87.2019.2444

\section{Resumen}

El objetivo de la investigación fue demostrar la relación entre la administración estratégica y la calidad en las micros, pequeñas y medianas empresas de la industria del vestido en Puebla, México. Esta investigación contribuye con conocimiento al reforzar la teoría según la cual la calidad es un problema de productividad y competitividad para las empresas. Los resultados que se muestran pertenecen a una investigación positivista, de revisión documental y de aplicación, con un estudio de campo sobre un modelo que permite determinar la relación entre la administración estratégica y la calidad en las empresas, respaldado por un coeficiente de correlación de 0,6500 y un nivel de significancia del $5 \%$. De forma práctica se usaron los conceptos de «calidad»y «administración estratégica» en la industria del vestido. Finalmente, el análisis de los resultados concluye que existe una relación positiva moderada entre la variable administración estratégica y la variable calidad en la industria del vestido en Puebla, pues queda claro que si se realiza un buen diagnóstico con un buen análisis interno y externo, que optimice estas variables de manera efectiva, será posible generar objetivos y estrategias que permitan mejorar la productividad y obtener ventajas competitivas frente a los otros mercados.

Palabras clave: administración estratégica; industria textil; industria del vestido; micros, pequeñas y medianas empresas; calidad; competitividad; productividad.

${ }^{1}$ Doctorado en Ciencias administrativas por el Instituto Politécnico Nacional. Magíster en Evaluación por la Benemérita Universidad Autónoma De Puebla. Ingeniero mecánico eléctrico (área industrial) por la Universidad Nacional Autónoma De México. ORCID: https://orcid.org/0000-0002-7143-3431

${ }^{2}$ Doctorado en Administración Gerencial por el Instituto de Estudios Universitarios. Magíster en mercadotecnia por el Instituto De Estudios Universitarios. Administrador de empresas de la Universidad Popular Autónoma del Estado De Puebla. ORCID: https:// orcid.org/0000-0003-1621-0806

${ }^{3}$ Doctorado en Administración gerencial por el Instituto De Estudios Universitarios. Magíster en Mercadotecnia por el Instituto De Estudios Universitarios. Administrador de empresas de la Benemérita Universidad Autónoma de Puebla. ORCID: https://orcid. org/0000-0002-7313-3723 


\section{Strategic management in the quality of the clothing industry in Puebla, Mexico}

\section{Abstract}

The aim of this research was to demonstrate the relationship between strategic management and quality in micro, small, and medium enterprises in the clothing industry in Puebla, Mexico. This research contributes knowledge by reinforcing the theory that quality is a productivity and competitiveness problem for companies. The results that are shown belong to a positivist research, of documentary review and application, with a field study on a model that allows to determine the relationship between strategic management and quality in the companies, supported by a coefficient of correlation of 0.6500 and a level of significance of $5 \%$. In a practical way, the concepts of "quality" and "strategic management" in the clothing industry were used. Finally, the analysis of the results concludes that there is a moderate positive relationship between the strategic management variable and the quality variable in the clothing industry in Puebla, since it is clear that if a good diagnosis is made with a good internal and external analysis that optimizes these variables effectively, it will be possible to generate objectives and strategies that allow to improve the productivity and competitive advantages over other markets.

Keywords: strategic management; textile industry; clothing industry; micro, small, and medium enterprises; quality; competitiveness; productivity.

\section{Gestão estratégica na qualidade da indústria do vestuário em Puebla, México}

\section{Resumo}

O objetivo da pesquisa foi demonstrar a relação entre gestão estratégica e qualidade em micro, pequenas e médias empresas da indústria de vestuário em Puebla, México. Esta pesquisa contribui com o conhecimento, reforçando a teoria de que a qualidade é um problema de produtividade e competitividade para as empresas. Os resultados apresentados pertencem a uma pesquisa positivista, de revisão documental e de aplicação, com um estudo de campo sobre um modelo que permite determinar a relação entre gestão estratégica e qualidade nas empresas, apoiada em um coeficiente de correlação de 0,6500 e um nível de significância de 5\%. Na prática, os conceitos de "qualidade" e "gestão estratégica" foram utilizados na indústria do vestuário. Por fim, a análise dos resultados conclui que existe uma relação positiva moderada entre a variável de gestão estratégica e a variável de qualidade na indústria de vestuário de Puebla, pois fica claro que, se um bom diagnóstico for feito com uma boa análise interna e externa, que se otimizam efetivamente essas variáveis, será possível gerar objetivos e estratégias que melhorem a produtividade e obtenham vantagens competitivas sobre outros mercados.

Palavras-chave: administração estratégica; indústria têxtil; indústria de roupas; micros, pequenas e médias empresas; qualidade; competitividade; produtividade. 


\section{Gestion stratégique de la qualité de la confection vestimentaire de Puebla, au Mexique}

\section{Résumé}

Lobjectif de cette recherche est de montrer la relation entre la gestion stratégique et la qualité des petites et moyennes entreprises de l'industrie du vêtement à Puebla, au Mexique. Cette investigation renforce la théorie selon laquelle la qualité est un problème de productivité et de compétitivité pour les entreprises. Les résultats présentés appartiennent à une recherche d’application positiviste et empirique constituant un modèle permettant de déterminer la relation entre gestion stratégique et qualité des entreprises favorisé par un coefficient de corrélation de 0,6500 et un niveau de signification de 5\%. Les concepts de «qualité» et de «gestion stratégique» dans l'industrie du vêtement ont également été pris en compte. Enfin, l'analyse des résultats conclut l'existence d'une relation positive et modérée entre la variable de gestion stratégique et la variable de qualité dans l'industrie du vêtement à Puebla et, en optimisant efficacement ces variables, il sera possible de générer des objectifs et des stratégies améliorant la productivité et créant des avantages compétitifs par rapport aux autres marchés.

Mots-clés: administration stratégique; industrie textile; industrie du vêtement; petites et moyennes entreprises; qualité; compétitivité; productivité 


\section{Introducción}

T a industria del vestido, en razón a su actividad, municipio de Puebla, por la generación de empleos y la contribución a la producción, entre otros factores. Las empresas que constituyen este sector son, en su mayoría, micros y pequeñas empresas que cuentan con un mayor uso intensivo de mano de obra y pueden entrar en un modelo de negocios en el que existe una capacidad de respuesta al cliente en relación con la moda, aunado al aumento de salario anual de la mano de obra de China. Sin embargo, ante una apertura comercial, este subsector de empresas se enfrenta a una competencia más fuerte y se deriva, entonces, la necesidad de mejorar su administración, sus áreas operativas y la calidad tanto de sus productos y servicios como de su gestión. Esta situación motiva a que el enfoque general del tema sea la administración estratégica en la calidad de las empresas.

Por tanto, esta investigación se sitúa en el contexto de la industria del vestido del Ayuntamiento de Puebla, porque es la alcaldía principal del Estado y allí se concentra la mayor parte de la manufactura de prendas de vestir, junto con otras dos industrias. Esto implica la existencia de fábricas dedicadas a la confección, entre las que se encuentran de diferentes tamaños, tales como micros, pequeñas, medianas y grandes empresas, en las que se elaboran diferentes productos como, por ejemplo, pantalones de mezclilla, batas, ropa de dama, ropa de bebé, ropa casual, calcetas, bermudas, blusas, faldas, playeras, camisetas y otros artículos. Además, se realizan mercancías por maquila y producción directa para exportación o consumo nacional, de manera que se manifiestan necesidades en las empresas a fin de lograr una mayor calidad y estar en capacidad de competir $y$, en consecuencia, de soluciones orientadas a satisfacerlas. En este caso, se considera la administración estratégica como una propuesta para influir en la calidad en las empresas.
En esta investigación se tomaron en cuenta los siguientes puntos: a) la pregunta general de la investigación: ¿qué relación existe entre la administración estratégica y la calidad en las empresas de prendas de vestir de la industria del vestido del municipio de Puebla?; b) el objetivo general O1: demostrar la relación entre la administración estratégica y la calidad en las empresas de prendas de vestir de la industria del vestido en el municipio de Puebla. Los objetivos específicos que se consideraron se enlistan a continuación:

- O2: indagar la relación entre la formulación de la estrategia de la empresa y la organización enfocada en el cliente, el enfoque en procesos y del sistema hacia la gestión, así como en el objetivo hacia la toma de decisiones.

- O3: comprobar la relación entre la implementación de la estrategia de la empresa, el liderazgo y la mejora continua, la participación de todo el personal y sus relaciones mutuamente benéficas con los proveedores.

- O4: explorar la relación entre la evaluación de la estrategia de la empresa y las normas y el control de calidad, la participación de todo el personal y sus relaciones mutuamente benéficas con los proveedores.

c) La hipótesis general se planteó de forma correlacional y es la siguiente:

- H1: la administración estratégica de la empresa tiene relación con la calidad en las empresas de prendas de vestir de la industria del vestido ubicadas en el municipio de Puebla.

Las hipótesis específicas que se tomaron en cuenta se enlistan a continuación:

- H2: la formulación de la estrategia de la empresa se relaciona con la organización enfocada en el cliente, el enfoque en procesos y del sistema hacia la gestión, así como en el objetivo hacia la toma de decisiones. 
- H3: la implementación de la estrategia de la empresa se relaciona con el liderazgo, la mejora continua, la participación de todo el personal y sus relaciones mutuamente benéficas con los proveedores.

- H4: la evaluación de la estrategia de la empresa se relaciona con las normas y el control de calidad, la participación de todo el personal y sus relaciones mutuamente benéficas con los proveedores.
La investigación se presenta en la siguiente estructura. Se desarrolla la revisión de la literatura, luego se expone el planteamiento y el uso de la metodología, se indican los resultados y la discusión de la investigación, para finalizar con la presentación de las conclusiones y las referencias. Se destacan en la sección de revisión de la literatura diferentes ponencias, la definición conceptual y operacional de las variables calidad y administración estratégica, sus correlaciones y la importancia de la industria del vestido.

\section{Revisión literaria}

A continuación, se describen una serie de aspectos relevantes encontrados en la literatura revisada.

\subsection{Calidad}

Los beneficios en las empresas que se obtienen por medio de la gestión de calidad son muy importantes, tal como se indica en la investigación de Carmona, Suárez, Calvo y Periánez (2016). Estos autores corroboran esta aseveración con análisis sobre el desarrollo de los sistemas de gestión de calidad en organizaciones del sur de España y el norte de Marruecos. Estas ventajas, entre otras, son: un mayor involucramiento del personal en la calidad, mejor comunicación y capacitación, calidad en los productos, servicio al cliente, procedimientos de trabajo, satisfacción del cliente y mayor fidelidad.

Otros estudios ratifican la aplicación en distintos ámbitos y los beneficios de los sistemas de gestión de calidad. Uno de ellos es el de Huerta, Sandoval, Preciado y Rodríguez (2017), el cual se aplicó en el campo de empresas de procesamiento de carne. La investigación se realizó mediante un análisis exploratorio con el fin de medir la utilización de los sistemas de calidad en un municipio del norte de México, y concluyó que con un sistema de gestión de calidad existe mayor desempeño en la producción y las ventas de productos de carne. Así mismo, en el ámbito de los servicios hospitalarios, Saturno et al. (2017) aportan la experiencia lograda a partir de los beneficios de la evaluación de las estrategias y los programas a nivel nacional e internacional para mejorar la calidad. Es decir, se puede medir la calidad en la atención a la salud, a través de la selección y la aplicación del conjunto de indicadores en hospitales públicos.

Con relación al concepto de calidad se encuentran pioneros como, por ejemplo, Joseph M. Juran, de Estados Unidos, o Kaoru Ishikawa, de Japón. Desde el enfoque de Juran (1990), entre distintos significados, la calidad es el producto de las características que logran la satisfacción del cliente seguido por su compra. Se concibe como «ausencia de deficiencias» al eliminar las quejas del cliente. Sobresale «la adecuación al uso al considerar clientes internos y externos».

Con relación a Ishikawa (1991), se destaca que, si se tiene conocimiento de los deseos del cliente, es posible controlar su satisfacción. Así, entonces, lo primero es conocer las necesidades de los clientes, conocer qué va a comprar y evitar probables reclamos. De esta manera, se puede llegar a que no se requiera una inspección a través de un adecuado y oportuno control. En este punto coincide con Juran, debido a que se contempla el control de la calidad, y lo define como «hacer lo que se debe hacer en todas las industrias» (Ishikawa, 1991). ¿Qué es el control 
de calidad?: La modalidad japonesa. Colombia: Norma. Al medir el resultado, existe control de calidad y de esta manera se obtienen ganancias en la empresa.

Con la globalización, los conceptos y los procedimientos orientados a la calidad se deben a las teorías que surgen, entre la década de los setenta y la de los ochenta del siglo XX, de los gurús y los líderes procedentes de los Estados Unidos, Japón, Inglaterra, Dinamarca y Suecia. Sobresalen Claus Moller, Phillip Crosby, Jan Carlzon y Shainín. Estos líderes, quienes realizaron importantes aportes, aunque con distintos enfoques, llegaron a la conclusión significativa de que los resultados se lograrán si la alta dirección de la empresa se implica con la calidad de manera constante (Montaudon, 2003).

Dado el éxito y los resultados que se han logrado en México y otros países con la metodología de las normas ISO 9000 de la Organización Internacional de Normalización ISO, esta se aplica hoy en empresas de diferentes sectores tanto privados como públicos. En especial, la norma ISO 9001:2000 que se aplica en empresas pequeñas, medianas y grandes, en las que, de manera permanente, se mejora siempre con el propósito de lograr la comprensión, el conocimiento y la coordinación de los directivos de mayor nivel sobre los principios de la gestión de calidad (Comité Nacional de Productividad e Innovación Tecnológica, A.C. [COMPITE, A.C.], 2003). Estos principios son: a) organización enfocada en el cliente: se puntualiza que la organización depende de sus clientes, en consecuencia, se deben compensar sus expectativas; b) liderazgo: los líderes se obligan a fundar y conservar el ambiente interno para que el personal se implique en los propósitos de la organización; c) involucramiento de todo el personal: el personal es el atributo de la organización y se demanda su total implicación a fin de cultivar sus capacidades, de modo que favorezcan a la empresa; d) enfoque en procesos: si las actividades se tramitan como un proceso se alcanzan resultados con mayor productividad; e) enfoque del sistema hacia la gestión: se puede lograr la eficacia y la eficiencia de una organización si se concibe y gestiona un proceso interrelacionado con miras a un objetivo que se fijó con anterioridad; f) mejora continua: es un objetivo constante de una organización; g) enfoque objetivo hacia la toma de decisiones: se logran los propósitos con la toma de decisiones y se realiza con base en el análisis de los datos y la información; y h) relaciones mutuamente benéficas con el proveedor: se crea valor si las relaciones con los proveedores son satisfactorias.

La aportación anterior se relaciona con la calidad total, lo cual supone una filosofía de gestión que indica la participación de todos los miembros de la organización en la búsqueda permanente de la superación y la mejora continua (Chiavenato, 2002). También concuerda que con calidad: la empresa lograría su nivel de excelencia y consigue satisfacer a sus clientes claves (Horovitz, 1995).

De lo anterior se percibe como característica esencial, en el propósito de lograr la calidad, que toda la organización debe funcionar como un sistema de gestión de calidad. De modo que deben estar interrelacionados la alta dirección y todo el personal. Así, conforme a los principios mencionados, se debe satisfacer al cliente $y$, en consecuencia, todas las actividades entran en una cadena que enlaza a los procesos, desde los proveedores hasta los clientes, e implica la compra de los productos de la empresa. Por tanto, las dimensiones y los indicadores se pueden determinar por medio de un sistema de gestión de calidad que formalice a la organización con un enfoque en el cliente y con base en sus principios.

En cuanto a la variable «calidad» como variable dependiente, se trata de entender por esta el logro de la completa satisfacción del cliente y que este lo puntualice y recompense con la compra de los productos de la organización, por medio de un sistema de gestión de calidad que formalice a la organización con enfoque en el cliente (Compite, 2003; Jurán 1990). A fin de soportar el desarrollo de la calidad como variable dependiente se considera la administración estratégica en su calidad de variable independiente y propuesta de solución, la cual en el transcurso del artículo se define y fundamenta. 
Tabla 1. Bases para la construcción teórica de la variable calidad

\begin{tabular}{|c|c|c|c|}
\hline \multicolumn{4}{|c|}{ Variable dependiente: calidad } \\
\hline \multicolumn{4}{|c|}{$\begin{array}{l}\text { Definición conceptual: se trata de lograr la completa satisfacción del cliente, y que este lo puntualice y recompense con la compra de } \\
\text { los productos de la organización, por medio de un sistema de gestión de calidad que formalice a la organización con enfoque en el } \\
\text { cliente (Compite, 2003; Jurán 1990). }\end{array}$} \\
\hline \multicolumn{4}{|c|}{$\begin{array}{l}\text { Definición operacional: está constituida por la organización enfocada en el cliente, el liderazgo, la participación de todo el personal, } \\
\text { el enfoque en procesos y del sistema hacia la gestión, la mejora continua, el enfoque objetivo hacia la toma de decisiones, las } \\
\text { relaciones mutuamente benéficas con el proveedor, las normas de calidad y el control de calidad. }\end{array}$} \\
\hline Dimensión & Indicador & Ítem & $\begin{array}{l}\text { Unidad de } \\
\text { medición }\end{array}$ \\
\hline $\begin{array}{c}\text { La organización } \\
\text { enfocada en el cliente }\end{array}$ & La organización & $\begin{array}{l}\text { 1. La organización siempre sabe de las necesidades presentes y } \\
\text { futuras de sus clientes, y las cumple. }\end{array}$ & \multirow{13}{*}{$\begin{array}{l}\text { 1) Totalmente en } \\
\text { desacuerdo } \\
\text { 2) En desacuerdo } \\
\text { 3) De acuerdo } \\
\text { 4) Totalmente de } \\
\text { acuerdo }\end{array}$} \\
\hline El liderazgo & La dirección & $\begin{array}{l}\text { 2. La dirección de la empresa se conoce por crear y guardar un } \\
\text { ambiente interno de motivación y reconocimientos para que } \\
\text { el personal participe de manera activa. }\end{array}$ & \\
\hline $\begin{array}{l}\text { La participación de } \\
\text { todo el personal }\end{array}$ & El personal & $\begin{array}{l}\text { 3. El personal siempre colabora en el logro de los objetivos de } \\
\text { la organización. }\end{array}$ & \\
\hline $\begin{array}{l}\text { El enfoque en procesos } \\
\text { y del sistema hacia la } \\
\text { gestión }\end{array}$ & Los procesos & $\begin{array}{l}\text { 4. La empresa se identifica porque sus actividades se ejecutan } \\
\text { mediante un proceso definido para el logro de la satisfacción } \\
\text { del cliente. }\end{array}$ & \\
\hline La mejora continua & Los objetivos & 5. Para la empresa la mejora continua es un objetivo intacto. & \\
\hline $\begin{array}{l}\text { El enfoque objetivo } \\
\text { hacia la toma de } \\
\text { decisiones }\end{array}$ & $\begin{array}{l}\text { La toma de } \\
\text { decisiones }\end{array}$ & $\begin{array}{l}\text { 6. En la empresa las decisiones siempre se ejecutan con base en } \\
\text { un análisis de datos y en la información. }\end{array}$ & \\
\hline $\begin{array}{l}\text { Relaciones } \\
\text { mutuamente benéficas } \\
\text { con el proveedor }\end{array}$ & Los proveedores & $\begin{array}{l}\text { 7. Las relaciones entre el proveedor y la empresa son buenas a } \\
\text { fin de fundar valor al cliente. }\end{array}$ & \\
\hline \multirow[b]{2}{*}{ Las normas de calidad } & El producto & $\begin{array}{l}\text { 8. Las normas de calidad del producto se encuentran por } \\
\text { escrito y se efectúan. }\end{array}$ & \\
\hline & $\begin{array}{l}\text { Las materias } \\
\text { primas }\end{array}$ & $\begin{array}{l}\text { 9. La empresa siempre atiende la calidad desde la selección de } \\
\text { los proveedores y poseen por escrito los requisitos de calidad } \\
\text { que deben cumplir las materias primas. }\end{array}$ & \\
\hline \multirow{4}{*}{ El control de calidad } & Los clientes & $\begin{array}{l}\text { 10. La empresa sabe que el nivel de quejas y devoluciones de } \\
\text { los clientes se hallan en los límites razonables. }\end{array}$ & \\
\hline & La comunicación & $\begin{array}{l}\text { 11. En la empresa siempre se reconocen todas las fallas en el } \\
\text { proceso total. }\end{array}$ & \\
\hline & La certificación & $\begin{array}{l}\text { 12. La empresa se identifica por contar con certificaciones } \\
\text { oficiales de calidad. }\end{array}$ & \\
\hline & Las herramientas & $\begin{array}{l}\text { 13. La empresa siempre usa la estadística y las gráficas para el } \\
\text { control de calidad. }\end{array}$ & \\
\hline
\end{tabular}

Nota. En la parte superior se presenta el nombre de la variable y sus definiciones conceptual y operacional. En las columnas se muestra su dimensión, el indicador, los ítems y la unidad de medición. En los renglones se exponen sus dimensiones, como, por ejemplo, la organización enfocada en el cliente, el liderazgo, la participación de todo el personal, el enfoque en procesos y del sistema hacia la gestión, la mejora continua, el enfoque objetivo hacia la toma de decisiones, las relaciones mutuamente benéficas con el proveedor, las normas de calidad y el control de calidad con sus correspondientes indicadores, con un total de 13 ítems, y como unidad de medición se tomaron en cuenta las siguientes alternativas: 1) totalmente en desacuerdo, 2) en desacuerdo, 3) de acuerdo, y 4) totalmente de acuerdo.

Fuente: Elaboración propia. 
Además, como parte de la revisión literaria se construyó la variable «calidad». Esta se compone de las dimensiones organización enfocada en el cliente, liderazgo, participación de todo el personal, enfoque en procesos y del sistema hacia la gestión, mejora continua, enfoque objetivo hacia la toma de decisiones, relaciones mutuamente benéficas con el proveedor, normas de calidad y control de calidad (Compite, 2003; Jurán 1990) (Véase la tabla 1).

Con relación a la construcción teórica de la tabla 1 se realizó con la finalidad de puntualizar la definición conceptual y operacional de la variable calidad. Se diseñó en consideración a los siguientes términos: dimensiones, esto es, aspectos integrantes de la variable; indicadores o guías del ítem; y sus ítems o partes de una prueba. Estos ítems forman la prueba resultante que se aplicó en las empresas de prendas de vestir.

\subsection{Administración estratégica}

Como un primer antecedente para construir este concepto se establece que la administración estratégica es un conjunto de estrategias organizacionales y de orientaciones administrativas que son claves en la toma de decisiones orientadas a resolver los problemas de la empresa. Esto al tomar en cuenta que la planeación estratégica se considera como administración estratégica, dado su desarrollo en la solución de los problemas en las organizaciones empresariales y el pensamiento administrativo que está ligado a estos conceptos (Alburquerke, Carrillo y Brabilla, 2013; Tovstiga, 2012). De hecho, según Michael Porter, a través de la planeación estratégica se suministra el pensamiento (Araya, 2017; Porter, 1997) y se logra una fotografía de la situación de las fortalezas, las debilidades, las amenazas y las oportunidades cuando se aplica en la empresa familiar. En este sentido, obtienen las estrategias necesarias para que la empresa continúe rumbo a su futuro.

Otro antecedente que permite definir el concepto de administración estratégica se presenta en la investigación de Vázquez y Covarruvias (2014). De acuerdo con estos autores, la planeación estratégica es una planeación a largo plazo para la obtención de objetivos en tiempo y forma. Esta se aplicó en el diseño de un plan a largo plazo en el desarrollo agrícola en el estado de Sonora como base de las estrategias para el valor agregado regional, con lo cual se demostró que esta planeación se puede aplicar en cualquier campo, con la salvedad de que no quede como una lista de requisitos, sino que se formalice en tiempo y forma para lograr los objetivos, tal como los señala Arriaga (2014) en una investigación sobre la declaración de los valores en empresas familiares.

De acuerdo con David (2013), Bateman y Snell (2001), y Lana (2008), la administración estratégica es una herramienta o un proceso con visión estratégica que se desarrolla de forma continua e interactiva para la empresa como un todo integrado con su ambiente a nivel de ciencia, en el cual se llevan a cabo los procesos de formulación, implementación y evaluación de las decisiones a fin de lograr los objetivos con eficiencia y eficacia. Además, coinciden con Thompson y Strickland (2004), quienes, adicionalmente, puntualizan que durante el proceso se pueden realizar los ajustes correctivos necesarios en la visión, los objetivos, la estrategia o su ejecución.

Se especifica que estos procesos son etapas de la administración estratégica (David, 2013), de tal manera que, mediante la administración estratégica, se logre la productividad de la empresa o la eficiencia y la eficacia de los objetivos que se propongan (Bateman y Snell, 2001; Koontz, Weihrich y Cannice, 2012).

La administración estratégica es un diagnóstico de la empresa en sus aspectos internos y externos con la finalidad de formular estrategias orientadas a lograr las ventajas competitivas (Torres, 2014). Se encuentra ligada a la cadena de valor, la cual permite analizar las actividades de la empresa e identificar sus ventajas competitivas, así como evaluar sus fuentes a fin de aumentar el margen de utilidad y valor para el cliente (Porter, 1997). Dentro de la cadena de valor, el concepto «administración» forma parte de las actividades secundarias que están interrelacionadas 
con las actividades primarias y son el soporte de ellas. Las actividades primarias son las actividades que tienen como objetivo el mayor margen de utilidad y valor para el cliente de forma directa, siempre y cuando se tenga un eficiente apoyo de las actividades secundarias. A fin de lograr estos objetivos es importante que el cliente esté satisfecho y se requiere que todas las actividades funcionen como un buen sistema de gestión de calidad.

Por lo que se refiere a la variable administración estratégica en cuanto variable independiente, se trata de entender por esta el proceso de formular, implementar y evaluar las decisiones con el fin de que la empresa consiga sus objetivos con eficiencia y eficacia (David, 2013).
Además, como parte de la revisión literaria, también se construyó la variable «administración estratégica», la cual integran las dimensiones formulación de la estrategia, implementación de la estrategia y evaluación de la estrategia (véase la tabla 2).

Con relación a la construcción teórica de la tabla 2 , se elaboró con el fin de detallar la definición conceptual y operacional de la variable administración estratégica. Se planteó en consideración a los siguientes términos: dimensiones, esto es, aspectos integrantes de la variable; indicadores o guías del ítem; y sus ítems, es decir, partes de una prueba. Estos ítems forman la prueba resultante que se aplicó en las empresas investigadas. 
Tabla 2. Bases para la construcción teórica de la variable administración estratégica

\begin{tabular}{|c|c|c|c|}
\hline \multicolumn{4}{|c|}{ Variable independiente: administración estratégica } \\
\hline \multicolumn{4}{|c|}{$\begin{array}{l}\text { Definición conceptual: es el proceso de formular, implementar y evaluar las decisiones con el fin de que la empresa consiga sus objetivos con } \\
\text { eficiencia y eficacia (David, 2013). }\end{array}$} \\
\hline \multicolumn{4}{|c|}{ Definición operacional: la conforman la formulación de la estrategia, la implementación de la estrategia y la evaluación de la estrategia. } \\
\hline Dimensión & Indicador & Ítem & Unidad de medición \\
\hline \multirow{8}{*}{$\begin{array}{l}\text { Dimensión } \\
\text { formulación de la } \\
\text { estrategia }\end{array}$} & Indicador misión & $\begin{array}{l}\text { 14. La empresa cuenta con definición de su misión por } \\
\text { escrito. }\end{array}$ & \multirow{17}{*}{$\begin{array}{l}\text { 4) Totalmente de acuerdo } \\
\text { 3) De acuerdo } \\
\text { 2) En desacuerdo } \\
\text { 1) Totalmente en } \\
\text { desacuerdo }\end{array}$} \\
\hline & Indicador personal & $\begin{array}{l}\text { 15. Todo el personal de mando intermedio trabaja en la } \\
\text { decisión de la planeación. }\end{array}$ & \\
\hline & $\begin{array}{l}\text { Indicador fuerzas y } \\
\text { debilidades }\end{array}$ & $\begin{array}{l}\text { 16. La empresa cuenta con definición de sus fuerzas y } \\
\text { debilidades. }\end{array}$ & \\
\hline & $\begin{array}{l}\text { Indicador amenazas y } \\
\text { oportunidades }\end{array}$ & $\begin{array}{l}\text { 17. La empresa tiene manifestadas sus amenazas y } \\
\text { oportunidades. }\end{array}$ & \\
\hline & $\begin{array}{l}\text { Indicador objetivos } \\
\text { generales }\end{array}$ & $\begin{array}{l}\text { 18. La empresa tiene instituidos sus objetivos a largo plazo } \\
\text { por escrito, admitidos por los miembros de la empresa. }\end{array}$ & \\
\hline & $\begin{array}{l}\text { Indicador objetivos } \\
\text { funcionales }\end{array}$ & $\begin{array}{l}\text { 19. La empresa tiene establecidos los objetivos por escrito } \\
\text { en sus áreas funcionales. }\end{array}$ & \\
\hline & Indicador estrategias & $\begin{array}{l}\text { 20. La empresa forja sus estrategias concretas para la } \\
\text { obtención de los objetivos planteados. }\end{array}$ & \\
\hline & Indicador recursos & 21. La empresa tiene definido cómo asignar sus recursos. & \\
\hline \multirow{8}{*}{$\begin{array}{l}\text { Dimensión } \\
\text { implementación } \\
\text { de la estrategia }\end{array}$} & Indicador eficacia & 22. La empresa siempre adquiere sus objetivos. & \\
\hline & Indicador eficiencia & $\begin{array}{l}\text { 23. La empresa siempre conquista sus objetivos con el } \\
\text { mínimo de recursos. }\end{array}$ & \\
\hline & $\begin{array}{l}\text { Indicador estructura } \\
\text { organizacional }\end{array}$ & 24. La empresa se basa en un organigrama. & \\
\hline & $\begin{array}{l}\text { Indicador descripción de } \\
\text { puestos }\end{array}$ & $\begin{array}{l}\text { 25. La empresa se basa con una descripción de puestos } \\
\text { por escrito. }\end{array}$ & \\
\hline & Indicador responsabilidad & $\begin{array}{l}\text { 26. La empresa se diferencia por tener notoriamente } \\
\text { definidas todas las responsabilidades. }\end{array}$ & \\
\hline & Indicador integración & $\begin{array}{l}\text { 27. Las áreas de personal y de compras ejecutan sus } \\
\text { actividades de forma eficiente. }\end{array}$ & \\
\hline & $\begin{array}{l}\text { Indicador toma de } \\
\text { decisiones }\end{array}$ & $\begin{array}{l}\text { 28. El empresario debe contar con la capacitación y la } \\
\text { experiencia adecuadas para motivar al personal en el } \\
\text { propósito de realizar las estrategias. }\end{array}$ & \\
\hline & Indicador comunicación & $\begin{array}{l}\text { 29. La empresa contiene canales de comunicación } \\
\text { adecuados para la toma de decisiones adecuadas. }\end{array}$ & \\
\hline $\begin{array}{l}\text { Dimensión } \\
\text { evaluación de la } \\
\text { estrategia }\end{array}$ & Indicador estándares & $\begin{array}{l}\text { 30. En la empresa siempre se controlan las estrategias y se } \\
\text { comprueban con estándares previamente fijados. }\end{array}$ & \\
\hline
\end{tabular}

Nota. En la parte superior se especifica el nombre de la variable y sus definiciones conceptual y operacional. En las columnas se describe su dimensión, el indicador, los ítems y la unidad de medición. En los renglones se exhiben sus dimensiones, como, por ejemplo, la formulación de la estrategia, la implementación de la estrategia y la evaluación de la estrategia, con sus correspondientes indicadores, con un total de 17 ítems. Como unidad de medición se emplearon las siguientes alternativas: 4) totalmente de acuerdo, 3) de acuerdo, 2) en desacuerdo, y 1) totalmente en desacuerdo.

Fuente. Elaboración propia. 


\subsection{Industria del vestido de Puebla}

En México, la industria del sector textil y del vestido tiene gran relevancia porque genera un mayor valor agregado y mayor número de empleos, y presenta una buena contribución a la producción en la industria manufacturera del país. Lo anterior, aunado a que se apoya a la maquila o la producción completa, ya que se emplea mano de obra de forma intensiva y con una mínima inversión por puesto de trabajo.

Esta industria contribuye a la exportación directa e indirecta, lo cual beneficia a México y otros países emergentes como generadores de moda. Aunque existen retos, también se encuentran oportunidades tales como el incremento del salario anual, del $13 \%$ y el $15 \%$ en China, en el rubro de la mano de obra. Por otra parte, existe un tipo de negocio llamado $\ll$ moda rápida» que le permite competir por su mayor creatividad y rapidez en la respuesta al cliente (Pérez y Rodríguez, 4 de octubre de 2015).

En el ámbito de la industria nacional y manufacturera, la industria textil y del vestido de México tuvo una participación del 0,4\% a nivel nacional y del 4,8\% de la industria manufacturera en el valor agregado bruto; del 1,1 \% nacional y el 11,1\% en la industria manufacturera con relación a personal ocupado; del $0,72 \%$ nacional y del 6,56 \% de la industria de manufactura en remuneración de asalariados. Se observa la importancia en este tipo de industria de la de prendas de vestir, ya que participó con el 52,7\% dentro de la estructura productiva de la industria textil y del vestido (Inegi, 2014).

El estado de Puebla ocupó el tercer lugar en la producción de la industria del vestido con un 8,5 \% de contribución (InegI, 2011), de modo que resultó la actividad de prendas de vestir como la más importante por su generación de empleos (Hernández, 12 de julio de 2017). A nivel municipal, el municipio de Puebla, junto a Teziutlán y Tehuacán generaron el $68 \%$ de la producción del estado de Puebla. (Inegi, 2011).

El estudio se realiza en empresas de tipo formal que corresponden a la confección de prendas de vestir dentro del municipio de Puebla, conforme a la clasificación de empresas de diferentes tipos marcados por Carstensen (2012).

Se resalta la importancia de la industria del vestido del municipio de Puebla, por lo cual aporta al estado de Puebla y a nivel nacional en los rubros de número de empleos, producción, valor agregado y oportunidades a nivel nacional e internacional, dada la flexibilidad en la producción y el modelo de negocios de $\ll$ moda rápida $»$.

\section{Metodología}

$S_{c}^{c}$ utilizó un enfoque, principalmente, cuantitativo, con un diseño no experimental, ya que no existe manipulación de la variable independiente. La investigación fue descriptiva y correlacional, dado un alcance en el que se detallan las variables y se busca una relación entre ellas. El método que se siguió fue deductivo, es decir, de lo general a lo particular; analítico, si se considera de un todo hacia las partes; y sintético para una explicación simplificada de estas. Las técnicas utilizadas fueron la encuesta y la entrevista. El instrumento fue un cuestionario con 30 ítems, utilizando la escala tipo Likert con las siguientes opciones: totalmente de acuerdo, de acuerdo, en desacuerdo, y totalmente en desacuerdo. La estrategia de aplicación fue seleccionar al directivo de la empresa seleccionada. El periodo en que se aplicó fue el primer trimestre del 2018.

La población fue de 358 empresas de prendas de vestir en el municipio de Puebla (InegI, 2011) y la muestra fue de 42 empresas, mediante la fórmula de muestra probabilística en dos pasos: 1) tamaño provisional de la muestra $\mathrm{n}^{\prime}=\mathrm{s} 2 / \mathrm{V}^{\wedge} 2$; y 2$)$ tamaño final de la muestra $n=n^{\prime} /\left(1+n^{\wedge} / N\right) \wedge$ (Hernández, Fernández y Baptista, 2006). La forma de elección 
de las empresas fue al azar de tipo tómbola. Las pruebas y las técnicas utilizadas en el análisis de los datos fueron: coeficiente de correlación de Pearson, coeficiente de determinación y coeficiente de alpha de Cronbach. Además, se utilizó el método de análisis de regresión lineal. El programa estadístico utilizado fue Minitab y el programa Excel.

La unidad de análisis utilizada fueron las empresas micros, pequeñas y medianas dentro del sector de prendas de vestir. De la encuesta de 42 empresas, el $57 \%$ fueron micros y pequeñas empresas. La microempresa - de 0 a 30 trabajadoresrepresentó el $24 \%$; la pequeña empresa —31 a 100 trabajadores- el $33 \%$ del total; la mediana - 101 a 500 trabajadores- el $21 \%$; y la gran empresa —más de 500 trabajadores- el $19 \%$. El giro y los productos específicos se componen de batas con el $2 \%$, batas de baño con el $2 \%$, batas y toalla con el
$2 \%$, jeans con el $5 \%$, ropa con el $2 \%$, boxers con el $2 \%$, uniformes con el $2 \%$, ropa para dama con el $2 \%$, ropa para bebé con el $2 \%$, pantalón de mezclilla con el $17 \%$, pantalón de mezclilla y gabardina con el $2 \%$, prenda de piel con el $2 \%$, ropa para bebé, niños, adultos y otros con el $2 \%$, ropa íntima para dama con el $5 \%$, ropa para dama y niña con el $2 \%$, short y ropa con el $2 \%$, ropa infantil con el $2 \%$, ropa casual con el $2 \%$, calcetas de algodón con el $2 \%$, calcetas y playeras con el $2 \%$, falda, pantalón, bermudas con el $2 \%$, pantalón, blusas y playeras con el $2 \%$, pantalón, vestido, falda, blusa con el $5 \%$, pantalón y falda con el $2 \%$, cobijas y ropa con el $2 \%$, playera de algodón con el $5 \%$, playeras tipo polo con el $2 \%$, playeras y camisas con el $2 \%$, pantalón con el $2 \%$, telas y camisetas con el $2 \%$.

En la tabla 3 se expone el resumen técnico de la investigación sobre la metodología utilizada. 
Tabla 3. Resumen técnico de la investigación

\begin{tabular}{|c|c|}
\hline Investigación & Enfoque cuantitativo, investigación: descriptiva, correlacional y transversal. \\
\hline Unidad de análisis & $\begin{array}{l}\text { Empresas micros, pequeñas y medianas dentro del sector de prendas de vestir. De la encuesta } \\
\text { de } 42 \text { empresas, el } 57 \% \text { fueron micros y pequeñas empresas. La microempresa - de } 0 \text { a } 30 \\
\text { trabajadores - representó el } 24 \% \text {; la pequeña empresa - } 31 \text { a } 100 \text { trabajadores-el } 33 \% \\
\text { del total; la mediana - } 101 \text { a } 500 \text { trabajadores - el } 21 \% \text {; y la gran empresa - más de } 500 \\
\text { trabajadores - el } 19 \% \text {. El giro y los productos específicos se componen de batas con el } 2 \% \text {, } \\
\text { batas de baño con el } 2 \% \text {, batas y toalla con el } 2 \% \text {, jeans con el } 5 \% \text {, ropa con el } 2 \% \text {, boxers } \\
\text { con el } 2 \% \text {, uniformes con el } 2 \% \text {, ropa para dama con el } 2 \% \text {, ropa para bebé con el } 2 \% \text {, } \\
\text { pantalón de mezclilla con el } 17 \% \text {, pantalón de mezclilla y gabardina con el } 2 \% \text {, prenda de } \\
\text { piel con el } 2 \% \text {, ropa para bebé, niños, adultos y otros con el } 2 \% \text {, ropa íntima para dama con } \\
\text { el } 5 \% \text {, ropa para dama y niña con el } 2 \% \text {, short y ropa con el } 2 \% \text {, ropa infantil con el } 2 \% \text {, } \\
\text { ropa casual con el } 2 \% \text {, calcetas de algodón con el } 2 \% \text {, calcetas y playeras con el } 2 \% \text {, falda, } \\
\text { pantalón, bermudas con el } 2 \% \text {, pantalón, blusas y playeras con el } 2 \% \text {, pantalón, vestido, } \\
\text { falda, blusa con el } 5 \% \text {, pantalón y falda con el } 2 \% \text {, cobijas y ropa con el } 2 \% \text {, playera de } \\
\text { algodón con el } 5 \% \text {, playeras tipo polo con el } 2 \% \text {, playeras y camisas con el } 2 \% \text {, pantalón con } \\
\text { el } 2 \% \text {, telas y camisetas con el } 2 \% \text {. }\end{array}$ \\
\hline Método & Deductivo, analítico y sintético. \\
\hline Técnica & Es encuesta y con entrevista. \\
\hline Instrumento & $\begin{array}{l}\text { Se usó un cuestionario con } 30 \text { ítems y se utilizó la escala tipo Likert con las siguientes } \\
\text { opciones: totalmente de acuerdo, de acuerdo, en desacuerdo, totalmente en desacuerdo. }\end{array}$ \\
\hline $\begin{array}{l}\text { La estrategia de } \\
\text { aplicación }\end{array}$ & $\begin{array}{l}\text { - ¿A quién?: de empresa seleccionada a directivo. } \\
\text { - ¿Dónde?: en la empresa que se seleccionó. } \\
\text { - ¿Cuándo?: en el primer trimestre del } 2018 .\end{array}$ \\
\hline Población objetivo & Un total de 358 empresas de prendas de vestir en el municipio de Puebla (Inegi, 2011). \\
\hline $\begin{array}{l}\text { La fórmula usada y el } \\
\text { tamaño de la muestra }\end{array}$ & $\begin{array}{l}\text { La fórmula muestra probabilística en dos pasos: 1) tamaño provisional de la muestra n'=; y } \\
\text { 2) tamaño final de la muestra } n=(\text { Hernández, Fernández, y Baptista, 2006). El tamaño de } \\
\text { la muestra fue de } 42 \text { empresas. }\end{array}$ \\
\hline $\begin{array}{l}\text { Pruebas y técnicas } \\
\text { utilizadas en el análisis } \\
\text { de los datos }\end{array}$ & $\begin{array}{l}\text { Las pruebas estadísticas paramétricas utilizadas son: coeficiente de correlación de Pearson, } \\
\text { coeficiente de determinación y coeficiente de alpha de Cronbach. Además, se utilizó el } \\
\text { método de análisis de regresión lineal. }\end{array}$ \\
\hline $\begin{array}{l}\text { Programa estadístico } \\
\text { utilizado }\end{array}$ & Minitab y Excel \\
\hline
\end{tabular}

Nota. En la Tabla 3 se presenta el diseño de la metodología que se utilizó conforme al problema de investigación que se tomó en cuenta. Se consideró el enfoque cuantitativo con un tipo de investigación descriptiva, correlacional y transversal. La unidad de análisis que se eligió fueron las micro, pequeñas y medianas empresas del sector de prendas de vestir. Como métodos de investigación se utilizaron deductivos, analíticos y sintéticos. Las técnicas de investigación que se manejaron fueron la encuesta y la entrevista, y se utilizó como instrumento de medición un cuestionario con 30 ítems y la escala tipo Likert con las siguientes alternativas: totalmente de acuerdo, de acuerdo, en desacuerdo, y totalmente en desacuerdo. Se trabajó con directivos o dueños de la empresa elegida durante el primer trimestre del 2018. La población de empresas en el municipio de Puebla fue de 358, y se consiguió una muestra de 42. Las técnicas/pruebas usadas en el análisis de los datos fueron las pruebas estadísticas paramétricas coeficiente de correlación de Pearson, coeficiente de determinación y coeficiente de alpha de Cronbach, los métodos de análisis de regresión lineal y se emplearon los programas estadísticos de Minitab y Excel.

Fuente. Elaboración propia. 
Se realizó un análisis de correlación de Pearson en el marco del análisis estadístico, el cual consiste en una prueba estadística para experimentar la relación entre dos variables medidas en un nivel por intervalos o de razón (Kumar, 2006). A fin de puntualizar su correcta interpretación de resultados, se utilizó la tabla de significados propuestos por Portus (1985) que se expone en la tabla 4.

Tabla 4. Interpretación de la escala del coeficiente de correlación de Pearson

\begin{tabular}{|c|c|c|}
\hline Resultado & Significa & Marca \\
\hline 0 & Correlación nula & \\
\hline 0,01 a 0,19 & Correlación positiva muy baja & $*$ \\
\hline 0,2 a 0,39 & Correlación positiva baja & ${ }^{* *}$ \\
\hline 0,4 a 0,69 & Correlación positiva moderada & $* * *$ \\
\hline 0,7 a 0,89 & Correlación positiva alta & $* * * *$ \\
\hline 0,9 a 0,99 & Correlación positiva muy alta & $* * * *$ \\
\hline 1 & Correlación positiva grande y perfecta & $* * * * *$ \\
\hline
\end{tabular}

Nota. En la Tabla 4 se muestran los valores y los significados para la interpretación de los resultados de los diferentes coeficientes de correlación de Pearson. En la primera columna se presentan los valores de los coeficientes. En la segunda columna se describen los diferentes significados que corresponden a cada uno de los valores. El intervalo de variación del coeficiente de Pearson es de -1 a 1 . El valor de -1 significa que hay una correlación inversa perfecta; el valor de 1 representa que existe una correlación directa perfecta. Los valores entre el valor de cero a -1 o a 1 tienen diferentes niveles de correlación, a excepción del valor de cero que simboliza que no hay correlación entre las variables.

Fuente. Portus, 1985.

Finalmente, con el fin de mostrar la relación entre la variable administración estratégica y la variable calidad en las micro, pequeñas y medianas empresas de la industria de prendas de vestir en el municipio de Puebla, se proyectaron las hipótesis expuestas en la introducción de este artículo para usar el método de correlación y observar la relación de las variables, además del método de regresión lineal con el fin de observar la causalidad entre ellas.

\section{Resultados y discusión}

Dn la tabla 5 se presentan los objetivos generales - $\mathrm{O} 1$ y los objetivos específicos - O2, O3 y O4-, así como las hipótesis tanto general o $\mathrm{H} 1$ como las de trabajo $-\mathrm{H} 2, \mathrm{H} 3$ y $\mathrm{H} 4-$, los resultados y los comentarios de estas. El desarrollo analítico se presenta de la siguiente forma: a) en la tabla 6, la matriz de correlación entre las variables administración estratégica y calidad, que son las variables principales del artículo; b) en la tabla 7 , la matriz de correlación entre las dimensiones, es decir, los aspectos integrantes de las variables; c) en la tabla 8 el análisis de regresión entre las variables; y d) en la tabla 9 el análisis de regresión entre las dimensiones —aspectos integrantes — de las variables. 
Tabla 5. Objetivos, hipótesis, resultados y comentarios

\begin{tabular}{|c|c|c|}
\hline Obje & & Con \\
\hline $\begin{array}{l}\text { O1: demostrar la relación entre la } \\
\text { administración estratégica de y la } \\
\text { calidad en las empresas de prendas } \\
\text { de vestir de la industria del vestido } \\
\text { ubicadas en el municipio de Puebla. } \\
\text { H1: la administración estratégica de la } \\
\text { empresa tiene relación con la calidad } \\
\text { en las empresas de prendas de vestir de } \\
\text { la industria del vestido ubicadas en el } \\
\text { municipio de Puebla. }\end{array}$ & $\begin{array}{l}\text { Coeficiente de Pearson igual a } 0,6500 \\
\text { Coeficiente de determinación igual a } 0,4220 \\
\text { La ecuación que relaciona a las variables es: } \\
\text { Calidad = } 19,2+0,441 \text { Administración } \\
\text { estratégica }\end{array}$ & $\begin{array}{l}\text { La administración estratégica explica el } 42,2 \% \\
\text { de la variación de la calidad. La correlación } \\
\text { entre las variables es positiva moderada y, por } \\
\text { tanto, se acepta } \mathrm{H} 1 \text {, con lo que demuestra } \\
\text { una relación de causalidad, en la que la } \\
\text { administración estratégica de la empresa incide } \\
\text { de forma directa y positiva en la calidad de las } \\
\text { empresas de prendas de vestir ubicadas en el } \\
\text { municipio de Puebla. }\end{array}$ \\
\hline $\begin{array}{l}\text { O2: indagar la relación entre la } \\
\text { formulación de la estrategia de la } \\
\text { empresa y la organización enfocada en } \\
\text { el cliente, el enfoque en procesos y del } \\
\text { sistema hacia la gestión, así como en el } \\
\text { objetivo hacia la toma de decisiones. } \\
\text { H2: La formulación de la estrategia } \\
\text { de la empresa se relaciona con la } \\
\text { organización enfocada en el cliente, } \\
\text { el enfoque en procesos y del sistema } \\
\text { hacia la gestión, así como en el objetivo } \\
\text { hacia la toma de decisiones. }\end{array}$ & $\begin{array}{l}\text { Coeficiente de Pearson igual a } 0,5460 \\
\text { Coeficiente de determinación igual a } 0,3170 \\
\text { La ecuación que relaciona a las variables es: } \\
\text { la organización enfocada en el cliente, el } \\
\text { enfoque en procesos y del sistema hacia la } \\
\text { gestión, así como al objetivo hacia la toma } \\
\text { de decisiones }=5,21+0,187 \text { Formulación }\end{array}$ & $\begin{array}{l}\text { explica el } 31,7 \% \text { de la variació } \\
\text { organización enfocada en el cliente, e } \\
\text { en procesos y del sistema hacia la } \\
\text { así como en el objetivo hacia la } \\
\text { decisiones. La correlación es positiva n } \\
\text { y, por tanto, se acepta } \mathrm{H} 2 \text {, lo que demu } \\
\text { relación de causalidad, en la que la for } \\
\text { de la estrategia de la empresa inci } \\
\text { organización enfocada en el cliente, el } \\
\text { en procesos y del sistema hacia la ge } \\
\text { como al objetivo hacia la toma de deci }\end{array}$ \\
\hline $\begin{array}{l}\text { O3: comprobar la relación entre la } \\
\text { implementación de la estrategia de } \\
\text { la empresa y el liderazgo y la mejora } \\
\text { continua, la participación de todo el } \\
\text { personal y sus relaciones mutuamente } \\
\text { benéficas con los proveedores. } \\
\text { H3: la implementación de la estrategia } \\
\text { de la empresa se relaciona con el } \\
\text { liderazgo, la mejora continua, la } \\
\text { participación de todo el personal y sus } \\
\text { relaciones mutuamente benéficas con } \\
\text { los proveedores. }\end{array}$ & $\begin{array}{l}\text { Coeficiente de Pearson igual a } 0,5460 \\
\text { Coeficiente de determinación igual a } 0,2980 \\
\text { La ecuación que relaciona a las variables } \\
\text { es: liderazgo, la mejora continua, la } \\
\text { participación de todo el personal y } \\
\text { sus relaciones mutuamente benéficas } \\
\text { con los proveedores }=7,22+0,255 \\
\text { Implementación }\end{array}$ & $\begin{array}{l}\text { La implementación de la estrategia de la } \\
\text { empresa explica el } 29,8 \% \text { de la variación del } \\
\text { liderazgo, la mejora continua, la participación } \\
\text { de todo el personalysus relaciones mutuamente } \\
\text { benéficas con los proveedores. La correlación } \\
\text { es positiva moderada y, por tanto, se acepta H3, } \\
\text { lo que demuestra una relación de causalidad, } \\
\text { en la que la implementación de la estrategia } \\
\text { de la empresa incide en su liderazgo, la mejora } \\
\text { continua, la participación de todo el personal } \\
\text { y sus relaciones mutuamente benéficas con los } \\
\text { proveedores. }\end{array}$ \\
\hline $\begin{array}{l}\text { O4: explorar la relación entre la } \\
\text { evaluación de la estrategia de la } \\
\text { empresa y las normas y el control de } \\
\text { calidad, la participación de todo el } \\
\text { personal y sus relaciones mutuamente } \\
\text { benéficas con los proveedores. } \\
\text { H4: la evaluación de la estrategia de la } \\
\text { empresa se relaciona con las normas y } \\
\text { el control de calidad, la participación } \\
\text { de todo el personal y sus relaciones } \\
\text { mutuamente benéficas con los } \\
\text { proveedores. }\end{array}$ & $\begin{array}{l}\text { Coeficiente de Pearson igual a } 0,4190 \\
\text { Coeficiente de determinación igual a } 0,1760 \\
\text { La ecuación que relaciona a las variables es: } \\
\text { Normas y control de calidad, participación } \\
\text { de todo el personal y sus relaciones } \\
\text { mutuamente benéficas con los proveedores } \\
=19,7+2,14 \text { Evaluación }\end{array}$ & $\begin{array}{l}\text { La evaluación de la estrategia de la empresa } \\
\text { explica el } 17,6 \% \text { de la variación de las normas } \\
\text { y el control de calidad, la participación de } \\
\text { todo el personal y sus relaciones mutuamente } \\
\text { benéficas con los proveedores. La correlación } \\
\text { es positiva moderada, por tanto, se acepta H4, } \\
\text { lo que demuestra una relación de causalidad, } \\
\text { en la que las normas y el control de calidad } \\
\text { inciden en la participación de todo el personal } \\
\text { y sus relaciones mutuamente benéficas con los } \\
\text { proveedores. }\end{array}$ \\
\hline
\end{tabular}

Nota. La tabla tiene tres columnas. En la primera se exponen las hipótesis generales y de trabajo para la investigación. En la segunda se muestran los resultados con las pruebas estadísticas — coeficiente de Pearson y de determinación-. Finalmente, la tercera indica los comentarios con relación a la interpretación de los indicadores y la opinión sobre la evidencia y la decisión sobre las hipótesis.

Fuente. Elaboración propia con base en la metodología usada. 
Tabla 6. Matriz de correlación entre las variables administración estratégica y calidad

\begin{tabular}{|c|c|}
\hline Variables & Calidad \\
\hline & 0,6500 \\
Administración estratégica & 0,000 \\
& $* * *$ \\
\hline
\end{tabular}

Fuente. Elaboración propia con base en la metodología usada.

La tabla 6 expone la correlación entre las variables administración estratégica y calidad, en la cual la administración estratégica es la variable independiente y la calidad es la variable dependiente. Acorde a la tabla 5, la correlación entre las variables es positiva moderada. Por tanto, se apoya la hipótesis H1 y manifiesta una asociación moderada y de forma directa.

Tabla 7. Matriz de correlaciones entre las dimensiones — aspectos integrantes— de las variables administración estratégica y calidad

\begin{tabular}{|c|c|c|c|}
\hline Dimensiones & Formulación & Implementación & Evaluación \\
\hline Implementación & $\begin{array}{l}0,842 \\
0,000 \\
* * * *\end{array}$ & & \\
\hline Evaluación & $\begin{array}{l}0,579 \\
0,000 \\
* * *\end{array}$ & $\begin{array}{l}0,507 \\
0,000 \\
* * *\end{array}$ & \\
\hline $\begin{array}{l}\text { La organización enfocada en el cliente, el enfoque en los } \\
\text { procesos y del sistema hacia la gestión, así como en el } \\
\text { objetivo hacia la toma de decisiones. }\end{array}$ & $\begin{array}{c}0,563 \\
0,000 \\
* * *\end{array}$ & $\begin{array}{l}0,514 \\
0,000 \\
* * *\end{array}$ & $\begin{array}{c}0,411 \\
0,007 \\
* * *\end{array}$ \\
\hline $\begin{array}{l}\text { El liderazgo, la mejora continua, la participación de todo } \\
\text { el personal y sus relaciones mutuamente benéficas con los } \\
\text { proveedores. }\end{array}$ & $\begin{array}{l}0,514 \\
0,001 \\
* * *\end{array}$ & $\begin{array}{l}0,546 \\
0,000 \\
* * *\end{array}$ & $\begin{array}{l}0,305 \\
0,049 \\
* *\end{array}$ \\
\hline $\begin{array}{l}\text { Las normas y el control de calidad, la participación de todo } \\
\text { el personal y sus relaciones mutuamente benéficas con los } \\
\text { proveedores. }\end{array}$ & $\begin{array}{l}0,512 \\
0,001 \\
* * *\end{array}$ & $\begin{array}{l}0,642 \\
0,000 \\
* * *\end{array}$ & $\begin{array}{l}0,419 \\
0,006 \\
* * *\end{array}$ \\
\hline \multicolumn{4}{|c|}{$\begin{array}{l}\text { El liderazgo, la mejora continua, la participación de todo el personal y sus relaciones mutuamente benéficas con } \\
\text { los proveedores. } \\
\text { La organización enfocada en el cliente, el enfoque en procesos y del sistema hacia la gestión, así como en el } \\
\text { objetivo hacia la toma de decisiones. }\end{array}$} \\
\hline $\begin{array}{l}\text { El liderazgo, la mejora continua, la participación de todo } \\
\text { el personal y sus relaciones mutuamente benéficas con los } \\
\text { proveedores. }\end{array}$ & $\begin{array}{c}0,767 \\
0,000 \\
* * * *\end{array}$ & & \\
\hline $\begin{array}{l}\text { Las normas y el control de calidad, la participación de todo } \\
\text { el personal y sus relaciones mutuamente benéficas con los } \\
\text { proveedores. }\end{array}$ & $\begin{array}{l}0,638 \\
0,000 \\
* * *\end{array}$ & $\begin{array}{l}0,733 \\
0,000 \\
* * * *\end{array}$ & \\
\hline
\end{tabular}

Nota. La tabla 7 enseña la matriz de correlaciones entre las dimensiones de las variables administración estratégica y calidad, en la cual la administración estratégica es la variable independiente y la calidad la variable dependiente. Conforme a la tabla 5, las correlaciones entre las dimensiones de las variables independiente y dependiente son distintas, en asociaciones positivas y directas. Existe evidencia a favor con niveles de correlación positiva moderada para todas las todas las hipótesis $\mathrm{H} 1, \mathrm{H} 2, \mathrm{H} 3$ y H4.

Fuente: Elaboración propia. 
Tabla 8. Regresión lineal de las variables calidad y administración estratégica

\begin{tabular}{|c|c|}
\hline Variables & Variable dependiente: calidad \\
\hline Variable independiente: administración estratégica & $\begin{array}{c}\text { Administración estratégica en } \\
\text { función de la calidad }\end{array}$ \\
\hline Valor de la constante o intercepción & 19,20 \\
\hline Valor del coeficiente de la variable & 0,441 \\
Valor T-estadístico y nivel de significancia & $5,240^{* *}$ \\
\hline & 0,4220 \\
\hline ADJ & 0,4070 \\
\hline F- estadístico & $29,19^{* *}$ \\
\hline
\end{tabular}

Nota. La tabla 8 presenta los resultados de la regresión para la ecuación Calidad $=19,2+0,441$ Administración estratégica. La calidad es la variable dependiente y la administración estratégica es la variable independiente. Se enseñan en los renglones: el nombre de la variable independiente; el valor de la constante o intercepción; el valor del coeficiente de la variable con su valor t-estadístico y nivel de significancia del $5 \%(* *)$; el coeficiente de determinación; el coeficiente de determinación ajustado; el F-estadístico y su nivel de significancia del $5 \%\left({ }^{*}\right)$. Se indican en las columnas: el nombre de la variable dependiente; la relación entre las variables; y en las columnas restantes los valores relacionados con cada renglón. De acuerdo con el análisis de regresión realizado se alcanzó un tipo de relación lineal entre las variables calidad en función de la administración estratégica. Fue posible lograr una ecuación de regresión que permite apreciar el valor de la calidad conforme a un valor seleccionado de la variable independiente administración estratégica y, de esta manera, se calcularon los valores de la tabla. Con base en el análisis de varianza Anova se computó el F-estadístico y el coeficiente de determinación para apoyar la evidencia de que la administración estratégica explica el 42,2 \% de la variación de la calidad con una significancia del $\left.5 \% .{ }^{* *}\right)$.

Fuente: Elaboración propia.

Tabla 9. Regresiones lineales de las dimensiones de las variables calidad y administración estratégica

\begin{tabular}{|c|c|c|c|}
\hline Variables & $\begin{array}{c}\text { Dimensión de variable } \\
\text { dependiente, esto es, } \\
\text { calidad: } \\
\text { la organización enfocada } \\
\text { en el cliente, el enfoque } \\
\text { en procesos y del sistema } \\
\text { hacia la gestión, así como } \\
\text { en el objetivo hacia la } \\
\text { toma de decisiones. }\end{array}$ & $\begin{array}{c}\text { Dimensión de variable } \\
\text { dependiente, esto es, } \\
\text { calidad: } \\
\text { el liderazgo, la mejora } \\
\text { continua, la participación } \\
\text { de todo el personal y sus } \\
\text { relaciones mutuamente } \\
\text { benéficas con los } \\
\text { proveedores. }\end{array}$ & $\begin{array}{c}\text { Dimensión de variable } \\
\text { dependiente, esto es, } \\
\text { calidad: } \\
\text { las normas y el control de } \\
\text { calidad, la participación } \\
\text { de todo el personal y sus } \\
\text { relaciones mutuamente } \\
\text { benéficas con los } \\
\text { proveedores. }\end{array}$ \\
\hline $\begin{array}{c}\text { Dimensiones de } \\
\text { variable independiente } \\
\text { administración estratégica: } \\
\text { - formulación de la } \\
\text { estrategia } \\
\text { - la implementación de la } \\
\text { estrategia } \\
\text { - la evaluación de la } \\
\text { estrategia }\end{array}$ & $\begin{array}{c}\text { La organización } \\
\text { enfocada en el cliente, } \\
\text { el enfoque en procesos } \\
\text { y del sistema hacia la } \\
\text { gestión, así como en el } \\
\text { objetivo hacia la toma } \\
\text { de decisiones en función } \\
\text { de la formulación de la } \\
\text { estrategia de la empresa. }\end{array}$ & $\begin{array}{l}\text { El liderazgo, la mejora } \\
\text { continua, la participación } \\
\text { de todo el personal y sus } \\
\text { relaciones mutuamente } \\
\text { benéficas con los } \\
\text { proveedores en función de } \\
\text { la implementación de la } \\
\text { estrategia de la empresa. }\end{array}$ & $\begin{array}{l}\text { Las normas y el control de } \\
\text { calidad, la participación } \\
\text { de todo el personal y sus } \\
\text { relaciones mutuamente } \\
\text { benéficas con los } \\
\text { proveedores en función } \\
\text { de la evaluación de la } \\
\text { estrategia de la empresa. }\end{array}$ \\
\hline $\begin{array}{l}\text { Valor de la constante o } \\
\text { intercepción: }\end{array}$ & 5,21 & 7,22 & 19,7 \\
\hline
\end{tabular}




\begin{tabular}{|c|c|c|c|}
\hline $\begin{array}{c}\text { Valor del coeficiente de la } \\
\text { variable } \\
\begin{array}{c}\text { Valor T-estadístico y nivel } \\
\text { de significancia: }\end{array}\end{array}$ & $\begin{array}{c}0,187 \\
4,31^{* *}\end{array}$ & 0,255 & 2,14 \\
\hline$:$ & 0,3170 & $0,12^{* *}$ & 0,1760 \\
\hline ADJ : & 0,3000 & 0,2810 & 0,1550 \\
\hline $\begin{array}{c}\text { F- estadístico y nivel de } \\
\text { significancia: }\end{array}$ & $18,55^{* *}$ & $16,99^{* *}$ & $8,53^{* *}$ \\
\hline
\end{tabular}

Nota. Los resultados de las regresiones entre las dimensiones de las variables calidad y administración estratégica se presentan en la tabla 9. Calidad es la variable dependiente y la administración estratégica es la variable independiente. Se revelan en los renglones por cada dimensión de la variable independiente los siguientes datos: el nombre de la dimensión de la variable independiente; el valor de la constante o intercepción; el valor del coeficiente de la variable con su valor T-estadístico y el nivel de significancia del $5 \%\left({ }^{* *}\right)$; el coeficiente de determinación; el coeficiente de determinación ajustado; el F-estadístico y su nivel de significancia del $5 \%(* *)$. Se anotan en las columnas los nombres de las dimensiones de la variable dependiente calidad. En la primera intersección de cada renglón y columna se muestra la relación entre las variables; en el resto, los valores relacionados con cada renglón. Con el uso del análisis de regresión se logró un tipo de relación lineal entre las variables calidad y la administración estratégica. En consecuencia, se constituyó la ecuación de regresión para estimar el valor de cada dimensión de la variable calidad en función de un valor seleccionado de la dimensión de la variable independiente administración estratégica, y de esta manera se computaron los valores de la tabla. El análisis de varianza Anova se empleó para calcular el F-estadístico y el coeficiente de determinación, y así constituir que las dimensiones de la administración estratégica explican en un porcentaje la variación de las dimensiones de calidad con un nivel de significancia del $5 \%\left({ }^{* *}\right)$. La tabla presenta los resultados de las siguientes ecuaciones de regresión: la organización enfocada al cliente, el enfoque a procesos y del sistema hacia la gestión, así como al objetivo hacia la toma de decisiones $=5,21+0,187$ Formulación; Liderazgo, la mejora continua, la participación de todo el personal y sus relaciones mutuamente benéficas con los proveedores $=7,22+0,255$ Implementación; Normas y control de calidad, participación de todo el personal y sus relaciones mutuamente benéficas con los proveedores $=19,7+2,14$ Evaluación.

Fuente. Elaboración propia.

Con los resultados que se obtuvieron y con la metodología aplicada se obtiene y responde a la pregunta de investigación sobre la relación entre la administración estratégica y la calidad en las empresas de prendas de vestir del municipio de Puebla. Se encontró evidencia a favor de la hipótesis H1, en la cual la administración estratégica sí tiene una relación directa y positiva con la calidad con un nivel moderado de asociación. También se comprobó que la administración incide en la calidad $y$, en efecto, existe causalidad. Por otra parte, se lograron evidencias a favor en todas las dimensiones seleccionadas, lo que implica una asociación moderada en las hipótesis H2, H3 y H4.

Como consecuencia del estudio se pueden ratificar las siguientes aseveraciones que se expusieron en la revisión de la literatura:

a) El sector de prendas de vestir es muy importante para México, en razón a los empleos que genera, entre otros factores, así como por la oportunidad de exportar y competir con países como, por ejemplo, China, dada su creatividad y rapidez en la respuesta al cliente. b) Las empresas necesitan resolver problemas con eficiencia y eficacia, de modo que la administración estratégica se convierte en una herramienta clave en la toma de decisiones que permite solucionarlos, razón por la cual se coincide con los autores Alburquerke et al. (2013) y Tovstiga (2012), a fin de identificar su situación real y obtener las estrategias que le permitan tener a las empresas una continuidad en el futuro, en especial en las empresas familiares (Araya, 2017; Porter, 1997).

c) Se coincide con Vázquez y Covarruvias (2014), y con Arriaga (2014), en que con sus estudios manifiestan las diferentes aplicaciones en los ámbitos industrial y agrícola, con la precisión de que es indispensable que se involucre a todo el personal en congruencia con los objetivos y los valores de la empresa.

d) La dimensión formulación de la estrategia de la empresa se corresponde e incurre con la organización enfocada en el cliente, el enfoque en procesos y del sistema hacia la gestión, así como en el objetivo hacia la toma de decisiones 
- $\mathrm{H} 2$ - con una asociación moderada, y se demuestra la causalidad entre las variables; en este sentido, se confirman las ponencias de David (2013), Bateman y Snell (2001), Koontz et al. (2012), y Lana (2008), debido a que la administración estratégica es una herramienta o un proceso con visión estratégica, el cual se realiza de forma continua y actúa como un todo integrado que lleva a cabo, como primera etapa, el proceso de formulación en la toma de decisiones a fin de lograr los objetivos.

e) La dimensión implementación de la estrategia de la empresa está relacionada e incide en el liderazgo, la mejora continua, la participación de todo el personal y sus relaciones mutuamente benéficas con los proveedores - $\mathrm{H} 3-$ con una asociación moderada, y se demostró la causalidad entre ellas. Esto concuerda con los planteamientos de Thompson y Strickland (2004) cuando señalan que durante el proceso se ajustan de manera progresiva las estrategias para no desviarse de la visión y los objetivos previamente definidos y alcanzar la productividad. Adicionalmente, es afín con los planteamientos de David (2013), porque se trata de la segunda etapa de la administración estratégica en la que se ejecuta lo definido en la formulación e interviene el liderazgo, la mejora y todo el personal que se involucra en el logro de los objetivos con eficiencia y eficacia. f) En la dimensión la evaluación de la estrategia de la empresa concuerda e incurre con las normas y el control de calidad, la participación de todo el personal y sus relaciones mutuamente benéficas con los proveedores - $\mathrm{H} 4-$ con una asociación moderada, y también tiene relación con lo que plantean David (2013), Koontz et al. (2012), Bateman y Snell (2001), y Torres (2014), porque es en el último proceso de la administración estratégica en el que se emiten los juicios de valor que permitan determinar si se está cumpliendo con los objetivos, con la eficiencia y la eficacia tanto de todo el personal como de los proveedores. De acuerdo con Porter (1997), todo esto se encuentra interrelacionado en una cadena de valor, en este caso se destacan las actividades de administración y de gestión de calidad, lo que incluye a los proveedores porque se considera que estos tienen su propia cadena de valor, la cual se une a la cadena de la empresa. De esta forma, si se realiza un buen diagnóstico con un buen análisis interno y externo será posible generar estrategias que permitan obtener ventajas competitivas.

Por último, de acuerdo con el soporte de la revisión de la literatura, los resultados ratifican la relación y la aplicación de la administración estratégica en la calidad a fin de obtener la productividad de las empresas del sector de prendas de vestir, en específico de las ubicadas en el municipio de Puebla. 


\section{Conclusiones}

C logra el objetivo del estudio con el tratamiento $\checkmark$ expuesto al contribuir con evidencia a favor de la hipótesis general $-\mathrm{H} 1$ - con el logro de una correlación positiva moderada, así como al promover el modelo de las variables administración estratégica y calidad. Con relación a las hipótesis de trabajo $\mathrm{H} 2$, $\mathrm{H} 3$ y $\mathrm{H} 4$ se evidenciaron con correlaciones positivas de nivel moderado.

A partir de los resultados alcanzados y comparados con la revisión de la literatura se confirman los siguientes puntos: a) a mejor calificación en la administración estratégica de la empresa, incidirá y conseguirá mejor calificación en la calidad de las empresas de prendas de vestir de la industria del vestido instaladas en el municipio de Puebla; b) a mejor calificación en la formulación de la estrategia de la empresa; incurrirá y adquirirá mejor calificación en la organización enfocada en el cliente, el enfoque en procesos y del sistema hacia la gestión, así como en el objetivo hacia la toma de decisiones de las prendas de vestir que se producen en el municipio de Puebla; c) a mejor calificación en la implementación de la empresa conseguirá mejor calificación en el liderazgo, la mejora continua, la participación de todo

\section{Referencias}

Alburquerke, A.; Carrillo, O.; Brabilla, A. (2013). El pensamiento estratégico: hacia una propuesta de agenda de investigación en los estudios organizacionales. Administración y Organizaciones, 30(2), 19-52.

Araya, A. (2017). Modelos de empresas familiares. Tec Empresarial, 11(1), 23-24. DOI: https://doi.org/10.18845/ te.v11i1.3093

Arriaga, A. (2014). Valores declarados en empresas familiares: ¿vivencia real operativa o solo requisito de la planeación estratégica? Anáhuac Journal, 11(1), 97-117.

Bateman, T.; Snell, S. (2001). Administración. Una ventaja competitiva ( $3^{\mathrm{a}}$ ed.). México: Mc Graw-Hill. el personal y sus relaciones mutuamente benéficas con los proveedores de las empresas de prendas de vestir ubicadas en el municipio de Puebla; d) a mejor calificación en la evaluación de la estrategia incurrirá y obtendrá mejor calificación en las normas y el control de calidad, la participación de todo el personal y sus relaciones mutuamente benéficas con los proveedores de las empresas de prendas de vestir ubicadas en el municipio de Puebla.

Con el proceso metodológico que se proyectó se lograron resultados coherentes. Se alcanzaron mediante los métodos de los análisis de correlación, determinación y regresión lineal que determinan la relación y la causalidad entre las variables.

Conforme a lo mostrado en la revisión de la literatura, el procesamiento y el análisis, se ratifica que con una buena administración estratégica es posible lograr buena calidad. Para finalizar, se sugieren como nuevas líneas de investigación el estudio y la aplicación de la relación entre las variables «administración estratégica» y la «calidad» a otras compañías de diferentes sectores de transformación, comerciales y de servicios, en otros municipios y estados de México, así como en el extranjero.

Carstensen, L. (2012). La maquila clandestina: el trabajo a domicilio informal en la industria textil y del vestido en Puebla. Bajo el Volcán, 3(15), 193-221.

Carmona, M.; Suárez, E.; Calvo, A.; Periánez, R. (2016). Sistemas de gestión de la calidad: un estudio en empresas del sur de España y norte de Marruecos. 22(1), 8-16. DOI: https:// doi.org/10.1016/j.iedee.2015.10.001

Chiavenato, I. (2002). Administración en los nuevos tiempos. Colombia: McGraw-Hill.

Comité Nacional de Productividad e Innovación Tecnológica, A.C. [Compite, A.C.] (2003). Introducción a los sistemas de gestión de la calidad ISO 9001:2000. México: Compite. 
David, F. (2013). Conceptos de administración estratégica (12a ed.). México: Pearson.

Hernández, M. (12 de julio de 2017). Textileras poblanas, sin ventas al exterior. El Economista. Recuperado de https://bit. ly/2w4aloR

Hernández, R., Fernández, C.; Baptista, P. (2006). Metodología de la investigación. México: McGraw-Hill.

Horovitz, J. (1995). La calidad del servicio. Colombia: McGraw-Hill.

Huerta, M.; Sandoval, S.; Preciado, M.; Rodríguez, J. (2017). Empresas cárnicas en el noroeste de México: análisis exploratorio en la medición de los sistemas de calidad y desempeño empresarial, Horizontes Empresariales, 16(1), 4-17.

Inegi. (2011). Estadísticas a propósito de la industria del vestido. Recuperado de https://bit.ly/3b2kd16

Inegi. (2014). La industria textil y del vestido en México 2014. Recuperado de https://bit.ly/2IVxNHu

Ishikawa, K. (1991). ¿Qué es el control de calidad?: la modalidad japonesa. Bogotá: Editorial Norma.

Juran, J. (1990). Juran y la planificación para la calidad. Madrid: Díaz de Santos.

Koontz, H.; Weihrich, H.; Cannice, M. (2012). Administración: una perspectiva global y empresarial (10 ${ }^{\mathrm{a}}$ ed.). México: McGraw-Hill.

Kumar, Y. (2006). Fundamental of search methodology and statics (7a ed.). Nueva Delhi: New Age International.

Lana, R. A. (2008). La administración estratégica como herramienta de gestión. Revista Cientifica «Visión de Futuro», 9(1). Recuperado de http://www.redalyc.org/ pdf/3579/357935469001.pdf
Montaudon, T. C. (2003). Historia de la calidad mundial y de los hombres que la hicieron posible (Tesis de maestría). Universidad Iberoamericana, México.

Pérez, L.; Rodríguez, A. (4 de octubre de 2015). La importancia del sector textil-vestido en México. Milenio. Recuperado de https://bit.ly/39Z1uDx

Porter, M. (1997). Ventaja competitiva: creación y sostenimiento de un desempeño superior. México: Cecsa.

Portus, L. (1985). Introducción a la estadística (2a ed.). Colombia: McGraw-Hill.

Saturno, P.; Martínez, I.; Poblano, O.; Vértiz, J.; Suárez, E.; Magaña, M.; Kagua, S. (2017). Implementación de indicadores de calidad de la atención en hospitales públicos de tercer nivel en México. Salud Pública de Méxixo, 59(3), 227-235. DOI: https://doi.org/10.21149/8228

Torres, Z. (2014). Administración estratégica. México: Grupo Editorial Patria.

Tovstiga, G. (2012). Estrategia en la práctica. La guía profesional para el pensamiento estratégico ( $2^{\mathrm{a}}$ ed.). Buenos Aires: Granica.

Thompson, A. A.; Strickland, A. J. (2004). Administración estratégica, textos y casos. México: McGraw-Hill.

Vázquez, M.; Covarruvias, J. (2014). Planeación estratégica con enfoque de megaplaneación como impulsor de desarrollo agrícola. Cultura Científica y Tecnológica, 11(54), 31-40. Recuperado de https://dialnet.unirioja.es/servlet/ articulo? codigo $=7082650$ 
